\title{
A computationally efficient method for self-planning uplink power control parameters in LTE
}

\author{
José Ángel Fernández-Segovia ${ }^{1 *}$, Salvador Luna-Ramírez ${ }^{1}$, Matías Toril $^{1}$, Ana Belén Vallejo-Mora
} and Carlos Úbeda ${ }^{2}$

\begin{abstract}
Uplink power control has a strong impact on the performance of mobile communication networks. In this work, an automatic parameter planning algorithm for the standardized power control scheme in the physical uplink shared channel (PUSCH) of long term evolution LTE is proposed. The method is conceived for the network design stage, when network measurements are still not available. The proposed heuristic algorithm can handle irregular scenarios at a low computational complexity. For this purpose, the parameter planning problem in a cell is formulated analytically through the combination of multiple regular scenarios built on a per-adjacency basis. Method assessment is carried out over a static system-level simulator implementing a real scenario. Results show that the proposed method can improve average user throughput and cell-edge user throughput when compared to current vendor approaches, which provide network-wide uniform parameter settings.
\end{abstract}

Keywords: Mobile network; Self-planning; LTE; Uplink power control; Real network sensitivity analysis

\section{Introduction}

Network planning plays a very important role in cellular communication networks. Before deployment, a thorough analysis must be done to achieve an adequate trade-off between capacity and coverage in the service area and predict future problems. A proper network planning avoids problems during the operational stage, minimizing (or, at least, delaying) subsequent capital investment [1,2].

Regardless of the radio access technology, cellular network planning is divided into core network planning and radio access network (RAN) planning. While the former is often only a dimensioning process, the latter entails the definition of RAN parameter settings that can be deployed in the roll-out stage. These parameters include not only physical parameters (e.g., site positions, antenna bearings, or power amplifier limits) but also logical parameters in radio resource management (RRM) algorithms [3-5]. In the past, due to the complexity of finding optimal settings for each specific scenario, vendors have provided

\footnotetext{
*Correspondence: jfs@ic.uma.es

1 Ingeniería de Comunicaciones, Universidad de Málaga, Campus de Teatinos S/N, 29071 Malaga, Spain

Full list of author information is available at the end of the article
}

operators with safe recommended values that should work reasonably well in most cases. Thus, the flexibility given by network parameters has not been fully exploited. To solve this situation, an effort has been made in the 3rd Generation Partnership Project (3GPP) long term evolution (LTE) standard to define the requirements for automating and improving network planning [6]. Likewise, automatic (or self-) planning has been identified by the industry as a key process in self-organizing networks (SON) [7-9].

Uplink power control (ULPC) is an important RRM process in mobile networks, as it has a direct impact on received signal and interference levels, as well as on user battery consumption. This makes ULPC an ideal candidate for automated parameter planning. In practice, the variability of propagation, traffic and interference conditions make it very difficult for operators to find optimal settings for these parameters before operation. For this reason, operators usually set ULPC parameters to safe values, which are deployed network wide. As a result, suboptimal network performance is obtained due to network irregularities. Even if this can be solved later during network operation, the provision of proper initial settings is greatly appreciated by operators.

\section{是 Springer}

(c) 2015 Fernández-Segovia et al: licensee Springer. This is an Open Access article distributed under the terms of the Creative Commons Attribution License (http://creativecommons.org/licenses/by/4.0), which permits unrestricted use, distribution, and reproduction in any medium, provided the original work is properly credited. 
In the literature, several power control schemes have been proposed. Fractional power control (FPC) has been selected for the physical uplink shared channel (PUSCH) in LTE $[10,11]$. A performance analysis of open-loop FPC is presented in $[12,13]$. Such an analysis is extended in [14-16] to closed-loop behavior. Later studies have evaluated more sophisticated power control schemes for LTE, which take into account interference and load conditions $[17,18]$. In [19], a parameter sensitivity analysis for the standardized FPC algorithm is carried out based on system-level simulations. As a result, a suboptimal parameter configuration is suggested for interferencelimited and noise-limited macro-cellular scenarios. However, finding the best parameter configuration for every cell in the network is a more challenging task, since the underlying problem is a large-scale non-separable nonlinear optimization problem. In [20], the adjustment of ULPC parameters in a single cell is formulated as a classical optimization problem with average throughput and cell throughput as alternative figures of merit. The latter analysis is extended to a multi-cell scenario in [21] by formulating ULPC as a non-cooperative game model. Then, a heuristic iterative optimization algorithm is proposed, where cells report UL power settings to the network management system and exchange power and interference information with their neighbor cells. In [22], a self-planning method is proposed for selecting the best parameter settings in FPC on a per-cell basis in an irregular LTE scenario. The proposal is based on an exhaustive search approach using Taguchi's method over a systemlevel simulator. A more computationally efficient planning method is presented in [23]. The core of the method is an analytical model that predicts the influence of the nominal power (a.k.a. power offset) and path-loss compensation factor on call acceptance probability for a given spatial user distribution. A suboptimal value of these parameters is computed on a per-cell basis by a randomized local greedy search algorithm. Alternatively, other studies consider tuning algorithms for the network operational stage. For instance, a self-tuning algorithm is proposed in [24] to dynamically adjust nominal power parameter based on overload indicator [25] so as to control the overall interference in the network. Similarly, in [26], a self-tuning algorithm for FPC is proposed based on fuzzy-reinforcement learning techniques. These self-tuning algorithms, conceived to be executed during network operation, can also be applied to network planning, provided that a system performance model is available. However, most self-tuning algorithms rely on iterations that require evaluating system performance for many different parameter settings. This iterative process is straight-forward for live networks due to the availability of performance measurements. However, this is not the case of network planning, where computations required to measure the quality of a new parameter plan at every iteration may jeopardize method scalability when large scenarios are considered. For this reason, most ULPC planning methods in the literature rely on simple analytical models for system performance assessment. To the authors' knowledge, few of them can handle irregular scenarios at a low computational cost and none of them considers closed-loop performance.

In this paper, a self-planning algorithm for ULPC parameters in the PUSCH of LTE is proposed. The selfplanning algorithm determines the maximum uplink cell load, $U_{U L}$, and the nominal PUSCH power parameter in FPC, $P_{0}$. Full path-loss compensation is assumed in this work (i.e., FPC parameter $\alpha$ is fixed to 1), as implemented in most first vendor releases. To deal with network irregularities, the parameter tuning problem is solved on a cell-by-cell basis by aggregating the results from multiple regularized scenarios built on a per-adjacency basis. Such a regularization approach reduces the size of the solution space, thus reducing the time complexity of the method. Both open-loop and closed-loop power control performance are considered in the problem model. Method assessment is carried out over a static systemlevel simulator implementing a real network scenario. The main contributions of this work are as follows: a) a thorough parameter sensitivity analysis of ULPC closedloop performance in a realistic simulated scenario and b) a self-planning method for ULPC parameters that can handle irregular scenarios at a low computational complexity and considers both open- and closed-loop ULPC performance. Similar to [23], the proposed heuristic algorithm can handle irregular scenarios while still using a semi-analytical approach. Unlike [23], the optimized parameters are the nominal power and the maximum cell load, instead of the path-loss compensation factor. More importantly, the method proposed here has better scalability and computationally efficiency due to the regularization approach. The rest of the paper is organized as follows. Section 2 outlines the LTE system model used in the planning process. Section 3 describes the design of the proposed planning algorithm. Section 4 presents simulation results obtained in a real network scenario. Finally, Section 5 presents the concluding remarks.

\section{Uplink system model}

LTE uplink comprises three physical channels: physical random access channel (PRACH), physical uplink shared channel (PUSCH), and physical uplink control channel (PUCCH) [27]. In particular, PUSCH is used to deliver user data and control information for active users in the uplink, which makes it an important component to be dimensioned and optimized. This section outlines how the main $\mathrm{PUSCH}$ features, namely the power control 
algorithm and the physical resource block (PRB) allocation scheme, are modeled in this work.

\subsection{Power control algorithm}

The aim of power control is to adjust the transmit power of the user equipment (UE) to fulfill quality of service (QoS) requirements at the base station (i.e., eNodeB, $\mathrm{eNB})$. The power control scheme for PUSCH combines an open-loop and a closed-loop algorithm. The former aims to compensate for slow channel variations, while the latter adapts to changes in the inter-cell interference conditions, or measurement and power amplifier errors. In the standardized algorithm [11], the UE transmit power (in $\mathrm{dBm}$ ) is given by

$P_{T X}=\min \{P_{t x_{\max },}, \underbrace{P_{0}+\alpha \cdot P L}_{\begin{array}{l}\text { basic open-loop } \\ \text { operating point }\end{array}}+\underbrace{\Delta_{T F}+f\left(\Delta_{T P C}\right)}_{\text {dynamic offset }}+\underbrace{10 \cdot \log _{10} M_{P U S C H}}_{\text {bandwidth factor }}\}$,

where $P_{t x_{\max }}$ is the maximum UE transmit power, $\alpha$ is the channel path-loss compensation factor (set to 1 in this work), $P L$ are the propagation losses, $M_{P U S C H}$ is the number of PRBs assigned to the UE, and $\Delta_{T F}+f\left(\Delta_{T P C}\right)$ is a dynamic term that depends on the selected modulation scheme and power-control commands sent by the eNB. Equation 1 consists of three parts: a basic open-loop operating point, a dynamic offset controlled by closedloop operation, and a bandwidth correction factor. The open-loop term consists of a semi-static level given by the parameter $P_{0}$ (known as nominal power), defining the average received signal level target for all UEs in a cell, and a path-loss compensation term, controlled by path-loss compensation factor, $\alpha$. From (1), it follows that setting $P_{0}$ controls UE transmit power, thus determining the perceived signal-to-interference-plus-noise ratio (SINR) in the serving cell and the interference level in surrounding cells.

\subsection{PRB allocation scheme}

The PRB allocation scheme also has a strong impact on UL performance. PRB allocation is done by a scheduler in the eNB, which assigns PRBs to users so as to maximize average cell throughput while keeping cell-edge user throughput within reasonable limits. For mathematical tractability, a simplified scheduling algorithm consisting of two stages is used in this work. It is assumed that a) full spectral sharing is used (i.e., frequency reuse is 1), b) radio resources are independently allocated between cells (i.e., PRB allocation is done on a cell basis), c) users are uniformly distributed in a cell, but unevenly distributed among cells, and d) any user has infinite data to transmit (i.e., full buffer service model). In a first stage, referred to as naive scheme, a preliminary PRB assignment is made based on the open-loop power control algorithm. Thus, an estimate of the maximum number of PRBs assigned for each user is obtained, from which PRB usage and average interference levels can be predicted. Then, a second scheme, referred to as refined scheme, considers the closed-loop power control algorithm. With the latter scheme, the final PRB assignment guaranteeing a minimum SINR is computed based on interference estimates with the PRB assignment suggested by the first naive scheme. Both schemes are described next.

\subsubsection{Naive scheme - interference estimation}

A theoretical PRB allocation scheme is first computed based on open-loop power control. A grid of positions in a cell is defined, so that each position represents a potential user. The number of PRBs assigned to user $k, M(k)$, is the maximum number of PRBs that can be assigned to the UE located at that point while still guaranteeing $P_{0}$ at the serving eNB, computed as

$M(k)=\max \left\{M_{\min }, \min \left(10^{\left(P_{t x_{\max }}-\alpha \cdot P L(k)-P_{0}(S(k))\right) / 10}, M_{\max }\right)\right\}$

where $M_{\min }$ and $M_{\max }$ are the minimum and maximum number of PRBs that can be assigned to the UE, defined as network wide settings, $P L(k)$ are propagation losses (including antenna gains and path loss) for user $k$, and $P_{0}(S(k))$ is the value of $P_{0}$ for the cell serving user $k, S(k)$. In this work, $M_{\min }=2$ and $M_{\max }$ ranges from 6 to 100 depending on system bandwidth [28]. The value of $M(k)$ obtained by (2) is used to compute the transmit power for each user, $P_{T X}(k)$, as in (1). Then, the UL interference level received at each eNB $i, I_{U L}(i)$, is calculated as the sum of the interference from every neighbor cell $j$ and thermal noise. The interference from each neighbor $j$ is calculated as the average received signal level from UEs in that neighbor, weighted by the uplink cell load, $U_{U L}(j) . U_{U L}(j)$ is given by the PRB utilization ratio (i.e., the number of used PRBs divided by the number of available PRBs for a period of time). Thus,

$I_{U L}(i)=N_{T H}+\sum_{\forall j \in N(i)}\left(U_{U L}(j) \cdot \frac{1}{N_{u}(j)} \cdot \sum_{\forall k \in A(j)} \frac{P_{T X}(k)}{P L(k, i)}\right)$

where $N_{T H}$ is the thermal noise $(-119.4 \mathrm{dBm}$ in this work), $N(i)$ is the set of neighbors of cell $i, A(j)$ is the set of users (i.e., points in the service area) of cell $j, N_{u}(j)$ is the number of users (locations) in the service area of $j$, and $P_{T X}(k)$ is the UE transmit power of user $k$. 


\subsubsection{Refined scheme - SINR estimation}

Based on estimated interference levels, a second and more accurate PRB allocation defines the maximum number of PRBs while still satisfying a minimum SINR threshold, computed as

$$
M(k)=\max \left\{M_{\min }, \min \left(10^{\left(P_{t x_{\max }}-\alpha \cdot P L(k)-I_{U L}(S(k))-S I N R_{t h}\right) / 10}, M_{\max }\right)\right\}
$$

where $I_{U L}(S(k))$ is the UL interference level in cell $S(k)$ and $\operatorname{SINR}_{t h}$ is the minimum SINR value to be satisfied $(-2.8 \mathrm{~dB}$ in this work [28]). Equation 4 shows how the number of PRBs assigned to a user changes with radio link conditions (i.e., $P L$ and $I_{U L}$ ). A user experiencing good radio conditions (i.e., low path-loss and low interference level) can be assigned all available PRBs, $M_{\max }$. However, as path-loss and/or interference increases, the number of PRBs allocated to the user is reduced in (4). This PRB reduction allows a higher transmission power per PRB, and thus, the minimum SINR threshold is fulfilled.

\subsection{Key performance indicators (KPI)}

Several indicators are calculated to check user and network performance with different ULPC parameter settings. The maximum throughput per PRB achieved by a user is obtained from SINR values by the truncated Shannon bound formula [29] as

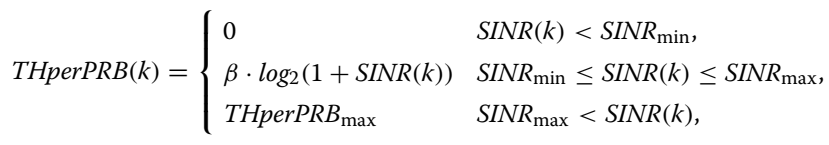

where THperPRB $B_{\max }$ is the maximum throughput per PRB achievable by the codeset, $S I N R_{\max }$ and $S I N R_{\min }$ are SINR values where maximum and minimum throughputs are reached, and $\beta$ is a constant. Then, the maximum achievable throughput is computed by multiplying throughput per PRB by the number of assigned PRBs obtained in (4). In this work, $T H$ perPR $B_{\max }=514 \mathrm{kbps}, S I N R_{\max }=14 \mathrm{~dB}$, $S I N R_{\min }=-9 \mathrm{~dB}$ and $\beta=0.6$.

Average user throughput, $T H_{\text {avg }}$, is used as a measure of cell capacity, while cell-edge user throughput, $T H_{c e}$, is used as a measure of cell coverage. Average user throughput is obtained as the average of individual user throughputs in the serving area of a cell, as

$$
T H_{\text {avg }}(i)=\frac{\sum_{\forall k \in A(i)} M(k) \cdot \operatorname{THperPRB}(k)}{N_{u}(i)} \cdot U_{U L}(i) .
$$

A user location, $k$, is assigned to cell $i$ if the latter is the one providing the minimum propagation losses. Cell-edge user throughput, $T H_{c e}(\mathrm{i})$, is defined as the fifth percentile of user throughput in the cell (i.e., the user throughput value exceeded by $95 \%$ of user locations in cell $i$ ).

\section{Self-planning method}

In this section, an algorithm for the automatic planning of the nominal power, $P_{0}$, and maximum UL cell load, $U_{U L}$, on a cell basis is presented. Firstly, a qualitative analysis of the influence of these parameters on network coverage and capacity is presented. Then, a preliminary parameter sensitivity analysis is performed in a regular scenario. From this quantitative analysis, the basic principles of the optimization algorithm are derived. The extension of the algorithm to deal with irregular scenarios is detailed later. With such an extension, ULPC settings can be adjusted on a per-cell basis to achieve better network performance.

\subsection{General considerations}

In mobile communication systems, coverage and capacity cannot be optimized independently as one affects the other. Network performance is a trade-off between cell-edge users, defining system coverage, and cell-center users, which have a larger influence on system capacity. This trade-off is the subject of the coverage-capacity optimization (CCO) problem, which has been identified as a relevant SON use case by 3GPP [30].

$\mathrm{CCO}$ can be performed by tuning ULPC parameters. Setting the average received signal level at the eNB, $P_{0}$, has a direct impact on the performance of both the adjusted cell and its surrounding cells. In the considered cell, a large value of $P_{0}$ enforces high transmit power for all users. Thus, users closer to the eNB should experience higher SINR values and, consequently, better UL performance. This is translated into higher peak user throughput in the cell at the expense of more user battery consumption. In contrast, cell-edge users that are power-limited cannot increase their transmit power when increasing $P_{0}$. At the same time, cell-edge users in surrounding cells experience low SINR due to increased interference level. Conversely, a low $P_{0}$ value favors cell-edge users in surrounding cells at the expense of cell-center users in the adjusted cell. Alternatively, UL cell load, $U_{U L}$, can be limited in a cell to avoid interference problems in surrounding cells. Control of $U_{U L}$ can be implemented by traffic management procedures, such as call admission control or cell load sharing.

The optimal $P_{0}$ and $U_{U L}$ settings in a cell are highly influenced by the propagation scenario and traffic distribution in both the cell under study and its neighbors. Therefore, any CCO algorithm must consider scenario irregularities and deal with neighbor cell conditions. As a consequence, $\mathrm{CCO}$ becomes a large scale non-separable multivariate optimization problem, where all cells must be jointly optimized. The size of the solution space is $\left(N_{v_{0}} \cdot N_{v_{U}}\right)^{N_{c}}$, where $N_{v_{P_{0}}}$ and $N_{v_{U}}$ are the number of possible values for $P_{0}$ and $U_{U L}$ parameters, and $N_{c}$ is the number of cells to be planned. The large size of the solution space prevents the use of exact algorithms, 
which are substituted by heuristic algorithms. To reduce the search space, some approaches only evaluate a limited set of representative parameter combinations, selected by, e.g., Taguchi's method [22]. Other approaches build an initial solution by assuming that all cells have the same parameter settings, which is then refined by a randomized local search process, e.g., greedy search [23] or simulated annealing. Even the latter approach requires many iterations to obtain solutions of reasonable quality, since any parameter change in a cell affects the performance of surrounding cells, and each iteration requires evaluating the overall network performance in the whole scenario after every parameter change.

In this work, a different approach is followed to reduce the search space. The global multi-variate optimization problem is divided into $N_{c}$ independent bi-variate optimization sub-problems (one per cell), each providing a sub-optimal solution for a cell $i, P_{0}(i)$ and $U_{U L}(i)$. To decouple sub-problems, some approximation is taken to model the irregular local scenario in the cell under study by a regular one. The idea is to approximate one real and complex scenario by many simple and regular scenarios that can be solved easily and independently. Apart from reducing the solution space, regularizing the scenario has other important advantages from the computational perspective: a) the grid resolution needed to obtain accurate propagation predictions is lower for regular scenarios than for irregular ones, and b) interference calculations for neighbor cells can be replicated due to the symmetry in a regular scenario, whereas these have to be treated individually in an irregular scenario due to the lack of symmetry. The following subsections describe first the optimization process in a regular scenario and later explain the extension to irregular scenarios.

\subsection{Regular scenario}

A preliminary sensitivity analysis shows the impact of tuning $P_{0}$ and $U_{U L}$ on network performance in a regular scenario. For this purpose, a static system-level simulator is used. The simulation scenario, shown in Figure 1, consists of one central tri-sectorized site surrounded by a first tier of tri-sectorized neighbor sites. Main simulation parameters are shown in Table 1. Performance statistics are collected only for the central cell $i$.

Network performance is evaluated for uniform $P_{0}$ and $U_{U L}$ settings in the scenario (i.e., all cells share the same parameter values). Figure 2 and Figure 3 show cell-edge and cell-average user throughput for cell $i, T H_{c e}(i)$ and $T H_{\text {avg }}(i)$, with different $P_{0}$ and $U_{U L}$ settings. Increasing $P_{0}$ leads to higher $P_{T X}(k)$ for cell-center users, thus increasing average user throughput. This improvement is limited by the interference from users in other cells, also increasing their $P_{T X}(k)$ in this regular scenario. Thus,

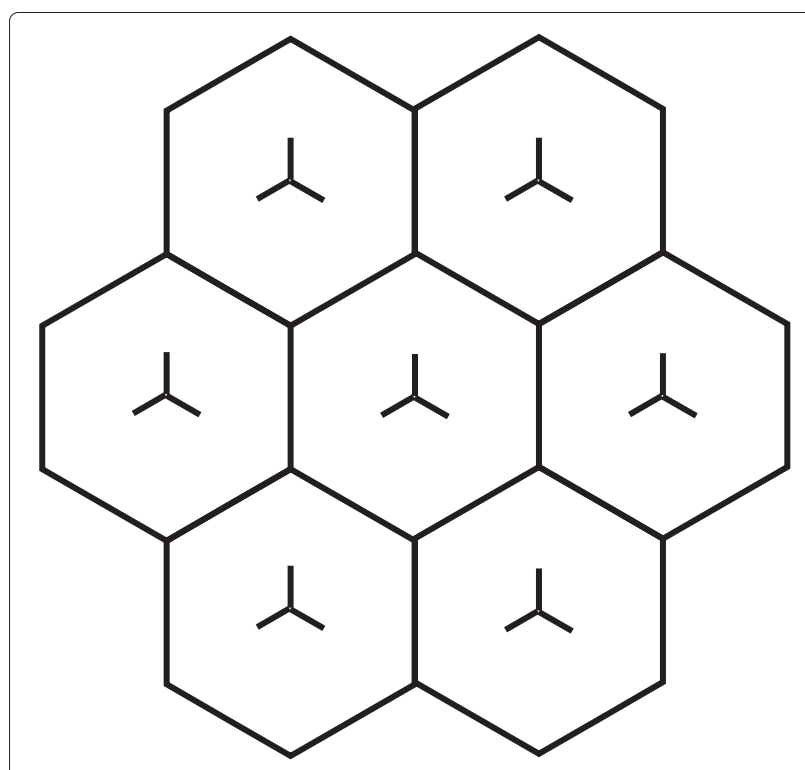

Figure 1 Regular scenario in the parameter sensitivity analysis.

$T H_{\text {avg }}(i)$ reaches a maximum value, after which $T H_{\text {avg }}(i)$ decreases. The largest value of $T H_{\text {avg }}(i)$ is obtained for high values of $P_{0}$ (i.e., $-102 \mathrm{dBm}$ ), where cell-center users can make the most of low propagation losses and received interference level is not much larger than the noise floor. In contrast, cell-edge performance quickly degrades as $P_{0}$ increases. This is due to the fact that cell-edge users reach their power limit, so that their transmit power does not change, but interference increases, as users in other cells increase their transmit power in the regular scenario. As a consequence, the maximum value for $T H_{c e}(i)$ is reached for low values of $P_{0}$, when $I_{U L}(i)$ is close to the noise floor

Table 1 Simulation parameters

\begin{tabular}{lc}
\hline Parameters & Settings \\
\hline Spectrum allocation & $10 \mathrm{MHz}(50 \mathrm{PRBs})$ \\
Carrier frequency & $2 \mathrm{GHz}$ \\
Cell layout & $7 \mathrm{eNBs}, 21$ sectors, regular grid \\
& $(200 \mathrm{~m}$ resolution). \\
Distance attenuation & $133.9+35.2 \log _{10}(d), d$ in $\mathrm{km}$ \\
Slow fading constant & $8 \mathrm{~dB}$ \\
Thermal noise density & $-174 \mathrm{dBm} / \mathrm{Hz}$ \\
Cell radius & $30 \mathrm{~m}$ \\
eNB antenna height & $5^{\circ}$ \\
eNB antenna tilt & $3 \mathrm{GPP}$ ideal [32] \\
eNB antenna pattern and gain & $23 \mathrm{kmm}$ inter-site distance) \\
Maximum UE transmit power & 1 (full compensation) \\
Path-loss compensation factor, $\alpha$ & Full buffer \\
Traffic model &
\end{tabular}




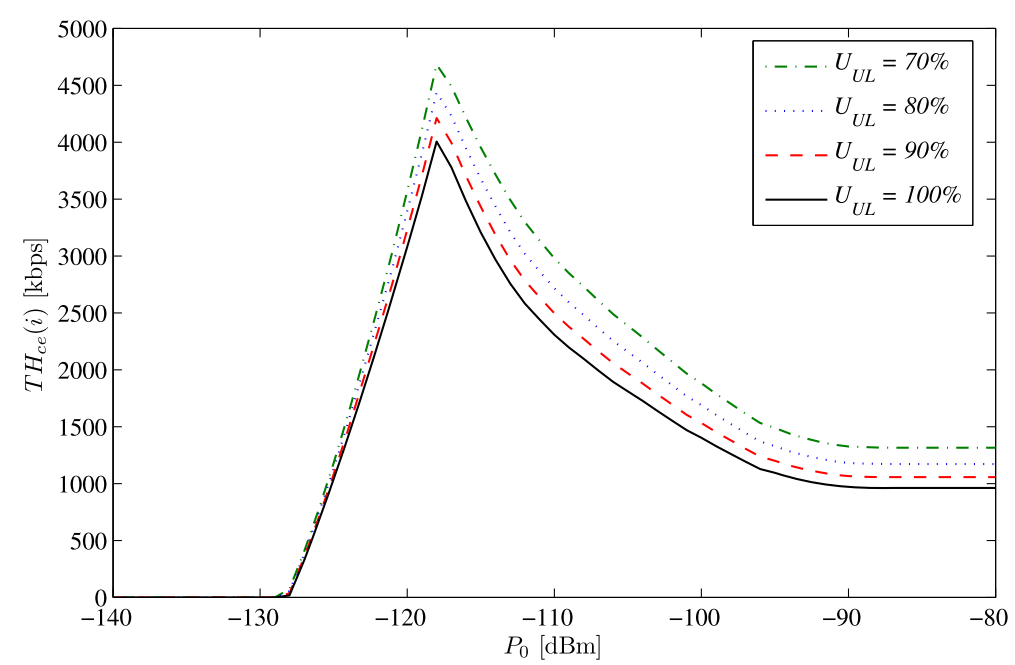

Figure 2 Cell-edge throughput performance in regular scenarios with uniform $P_{0}$ and $U_{U L}$.

(i.e., $-119.4 \mathrm{dBm})$. Note that small $P_{0}$ deviations from the optimal value cause large deviations in $T H_{c e}(i)$, but nearoptimal performance in $T H_{\text {avg }}(i)$. This is evidence that the trade-off between coverage and capacity is more critical for cell-edge users.

Based on the behavior observed in Figure 2 and Figure 3, a self-planning algorithm for $P_{0}$ and $U_{U L}$ in a regular scenario is designed. The flow diagram of the proposed iterative algorithm is shown in Figure 4. $P_{0}^{(n)}$ and $U_{U L}^{(n)}$ denote parameter values in iteration $n$. The optimization engine is a simple gradient-based local optimization method, whose aim is to maximize the average user throughput, $T H_{\text {avg }}(i)$, while keeping cell-edge throughput over a minimum, $T H_{c e}(i)>T H_{c e, \text { min }}$. Such a formulation of the CCO problem can be found in the literature (e.g., [31]). For this purpose, $P_{0}$ and $U_{U L}$ are initially configured to arbitrarily high values (e.g., $-80 \mathrm{dBm}$ and $100 \%$, respectively). Then, the algorithm iteratively decreases parameter values. In a first stage, when minimum cell-edge throughput is not fulfilled (i.e., $T H_{c e}(i)<T H_{c e, \min }$ ) and maximum $\mathrm{TH}_{c e}(\mathrm{i})$ value has not been reached, the optimization engine tries to increase $T H_{c e}(i)$ by decreasing $P_{0}$. Once coverage constraints are fulfilled (i.e., $T H_{c e}(i) \geq T H_{c e, \text { min }}$ ), the optimization engine keeps decreasing $P_{0}$ with the aim of maximizing $T H_{a v g}$, until $T H_{a v g}(i)$ starts to decrease (i.e., optimal $P_{0}$ in terms of $T H_{\text {avg }}(i)$ has been surpassed) or cell-edge throughput has become less than the minimum threshold. $U_{U L}$ is only decreased when minimum cell-edge throughput is not fulfilled. This is the case of interference-limited scenarios, consisting of very small

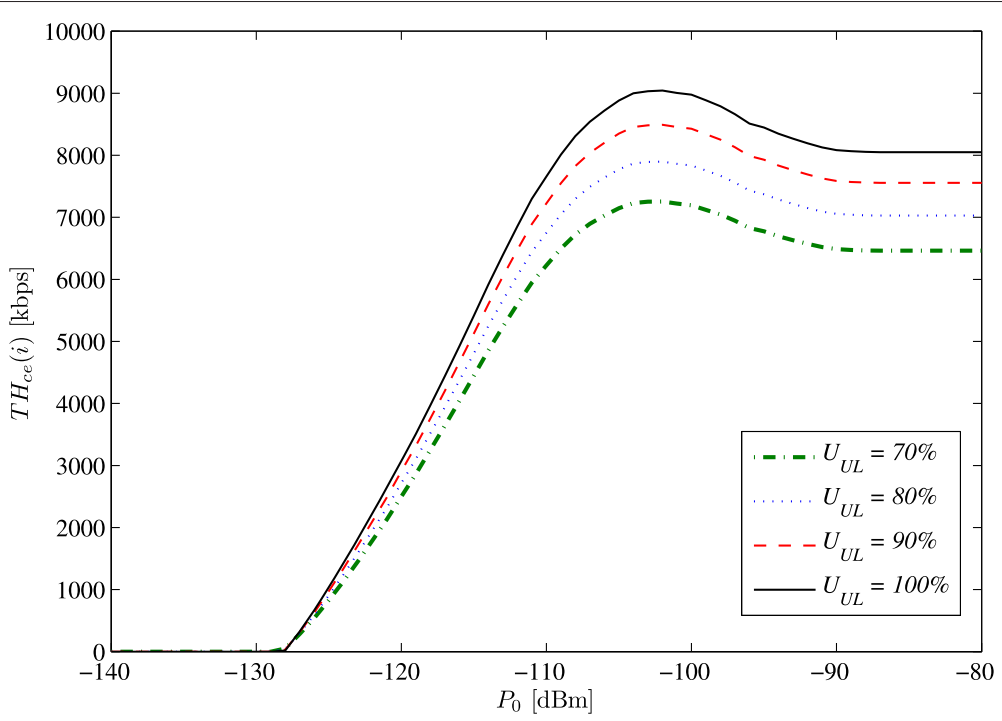

Figure 3 Average user throughput performance in regular scenarios with uniform $P_{0}$ and $U_{U L}$. 


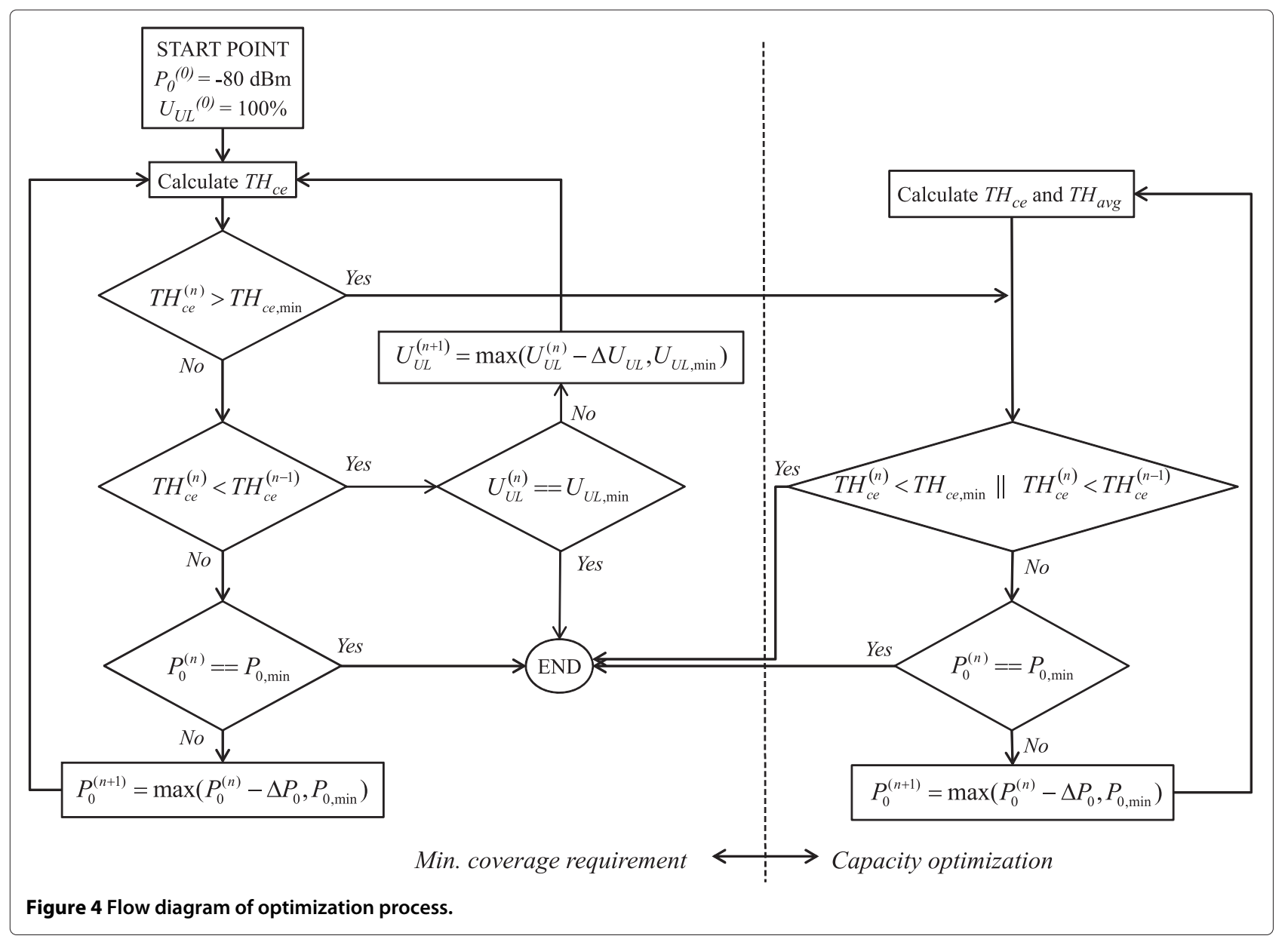

cells. Reducing $U_{U L}$ only when strictly needed is in line with operator policies, which only consider limiting the average PRB utilization in a cell under critical interference situations.

\subsection{Irregular scenario}

The above-described optimization algorithm is designed for a regular scenario, resulting in a solution where all cells have the same value of $P_{0}$ and $U_{U L}$. However, in live networks, propagation, traffic, and interference conditions greatly vary among cells. These irregularities translate into uneven parameter configuration for optimal network performance. The following paragraphs describe a method to deal with such irregularities. The method consists of four stages, namely global scenario construction, division into local regular scenarios, local problem solving, and aggregation of local solutions.

\subsubsection{Global scenario construction - adjacency definition}

The method starts by collecting network configuration data, consisting of site locations, antenna height, and antenna bearings (i.e., azimuth and tilt). This data is included in propagation models to define a list of relevant non co-sited neighbor cells for every cell in the scenario. Such a list is used later for regularization of local scenarios, as will be explained later.

Several options could be used to automatically define neighbors for every cell in the scenario. Relevant neighbors are usually identified by computing the contribution that every single user in each candidate neighbor cell has on the UL interference of the cell under study. In the absence of real measurements, this calculation requires computing a point-by-point path loss matrix, from which to identify cell dominance areas, which increases the computational load of the method.

For computational efficiency, a simple automatic neighbor definition algorithm is used here with planning purposes. The aim is to rank non co-sited neighbors by relevance assuming that the average path loss of all users in a neighbor cell is similar to that from neighbor eNB location. The number of neighbors must be kept within reasonable limits. An intuitive indicator for neighbor relevance in the $\mathrm{UL}, N R_{U L}$, is defined as

$$
N R_{U L}(i, j)=L(i, j)-A_{H}(i, j)-A_{V}(i, j)
$$


where $L(i, j)$ is the path loss between the cell under study $i$ and cell $j$ computed by some propagation model, and $A_{h}(i, j)$ and $A_{v}(i, j)$ are the horizontal and vertical gains for the antenna of cell $i$ to the location of eNB $j$. Roughly, the closest eNBs $j$ in terms of electrical distance tend to give the lowest $N R_{U L}(i, j)$ values and are labeled as relevant neighbors of cell $i$ (i.e., $j \in N_{r}(i)$, where $N_{r}(i)$ is the list of relevant non co-sited neighbors of cell $i$ ). To limit the number of relevant neighbors, neighbors $j$ having $N R_{U L} 10$ $\mathrm{dB}$ larger than that of the most relevant neighbor are discarded. For the same reason, a maximum of 12 neighbors per cell is configured.

\subsubsection{Division into local regular scenarios}

Once neighbors have been defined for each cell, the optimization process can start. Global network optimization is broken down into $N_{c}$ local optimization sub-problems, which are solved independently. Every sub-problem is solved by the regularization of one or several local scenarios. Two alternatives are considered, depending on how the regular local scenario is built for a cell $i$ :

- Mean radius approximation (MRA): A regular scenario, as that in Figure 1, is built for the cell under study $i$. Inter-site distance (ISD) in the regular scenario is taken from the global scenario by averaging ISDs between the site of cell $i$ and the site of its neighbors in the real irregular scenario, excluding co-sited cells. Thus, MRA assumes a single 'average' regular scenario per cell. Then, optimal $P_{0}(i)$ and $U_{U L}(i)$ values for cell $i$ are obtained by applying the optimization method described in Figure 4 to this equivalent regular scenario.

- Adjacency-based approximation (AA): The optimization problem for cell $i$ is broken down into $N_{\text {neigh }}(i)$ sub-problems, where $N_{\text {neigh }}(i)$ is the number of non co-sited neighbors of cell $i$ (i.e., the length of $N_{r}(i)$ ). For each pair of cells $(i, j)$, where $i$ is the optimized cell and $j$ is the selected neighbor, a regular scenario is built, as shown in Figure 5. ISD in the scenario is the one between cells $i$ and $j$ in the real network. In the figure, it is observed how the relative position (i.e., distance and antenna bearing angles) of cells in the real network is maintained. As a result of problem division, $N_{\text {neigh }}(i)$ regular scenarios are built and solved for each cell $i$.

Note that MRA needs a single optimization process per cell, whereas AA requires $N_{\text {neigh }}(i)$ optimization processes (one per neighbor). As a result, $N_{\text {neigh }}(i)$ pairs of sub-optimal $P_{0}$ and $U_{U L}$ values are obtained for each cell $i,\left(P_{0}(i, j), U_{U L}(i, j)\right)$. Some aggregation process is then needed to obtain a single solution per cell, $\left(P_{0}(i), U_{U L}(i)\right)$.

\subsubsection{Aggregation of adjacency-level solutions in $A A$}

A single value of $\left(P_{0}(i), U_{U L}(i)\right)$ is computed from all sub-optimal solutions involving cell $i$, where cell $i$ is either source or target in the adjacency, i.e., $\left(P_{0}(i, j)\right.$, $\left.U_{U L}(i, j)\right)$ and $\left(P_{0}(l, i), U_{U L}(l, i)\right)$. Four alternative aggregation criteria are defined, namely maximum, mean, minimum, and mixed. In each criterion, the final solution per cell is computed from adjacency-level solutions as follows:

- Maximum method (MaM):

$P_{0}(i)=\max \left\{\left[P_{0}(i, j) P_{0}(l, i)\right]\right\}, \quad \forall j \in N_{r}(i), l / i \in N_{r}(l)$

$U_{U L}(i)=\max \left\{\left[U_{U L}(i, j) U_{U L}(l, i)\right]\right\}, \quad \forall j \in N_{r}(i), l / i \in N_{r}(l)$

- Mean method (MeM):

$P_{0}(i)=$ mean $\left\{\left[P_{0}(i, j) P_{0}(l, i)\right]\right\}, \quad \forall j \in N_{r}(i), l / i \in N_{r}(l)$

$U_{U L}(i)=$ mean $\left\{\left[U_{U L}(i, j) U_{U L}(l, i)\right]\right\}, \quad \forall j \in N_{r}(i), l / i \in N_{r}(l)$

- Minimum method (MiM):

$P_{0}(i)=\min \left\{\left[P_{0}(i, j) P_{0}(l, i)\right]\right\}, \quad \forall j \in N_{r}(i), l / i \in N_{r}(l)$

$U_{U L}(i)=\min \left\{\left[U_{U L}(i, j) U_{U L}(l, i)\right]\right\}, \quad \forall j \in N_{r}(i), l / i \in N_{r}(l)$

- Mixed method (MxM). This approach uses the maximum criterion for $P_{0}(i)$, defined in (8), and the minimum criterion for $U_{U L}$, defined in (13). The aim is to maximize SINR by selecting a large $P_{0}$ value, even if interference is thus increased. Then, the lowest $U_{U L}$ is selected to mitigate such an interference increase.

Figure 6 summarizes the whole optimization process for a cell $i$ in the AA method, including regularization on a per-adjacency basis, sub-problem solving and solution aggregation.

\subsection{Computational complexity}

The time complexity of the MRA self-planning algorithm is $\mathrm{O}\left(N_{c}\right)$, since the number of sub-problems to be solved is the number of cells in the network. All of these subproblems deal with a regular scenario, which can be solved by a simple gradient search method on a single variable. 


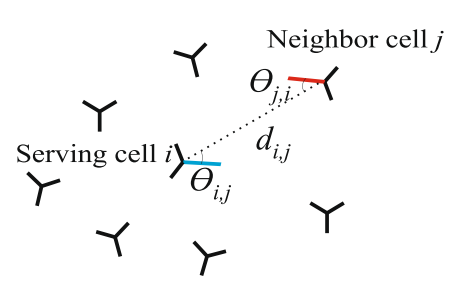

a) Irregular scenario

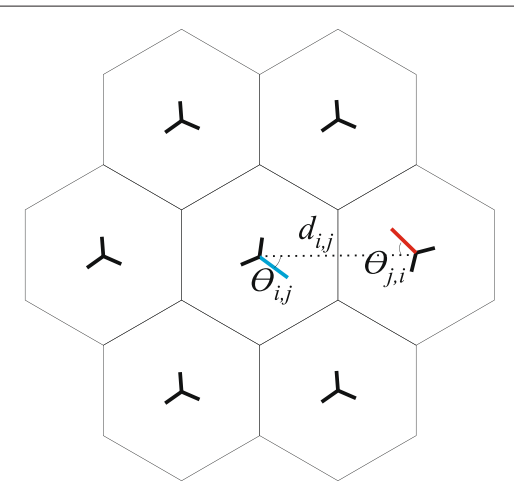

b) Regularized scenario

Figure 5 Scenario regularization. (a) Irregular scenario and (b) regularized scenario.

The time complexity of the AA self-planning algorithm is also $\mathrm{O}\left(N_{c}\right)$, since the number of sub-problems grows linear with the total number of relevant non co-sited neighbors in the scenario, which is proportional to the number of cells in the network.

\section{Performance analysis}

The solutions of the different variants of the proposed algorithm are tested in a system-level simulator implementing a real irregular scenario. The simulator includes all UL functionalities described in Section 2. For clarity, the analysis set-up is described first and results are presented later.

\subsection{Analysis setup}

The considered scenario consists of 233 sites (699 cells) in a large metropolitan area. Location, azimuth, and antenna tilts are obtained from live network configuration data. ISDs range from 0.3 to $6 \mathrm{~km}$. Other simulation parameters are shown in Table 1.

Five different self-planning approaches are tested. A first method uses MRA with neighbor cell selection as described in Section 3.3.1 (referred to as MRA). The other four methods use AA for dealing scenario irregularities, considering MaM, MeM, MiM, and MxM as solution aggregation criterion, respectively. To quantify the benefit of tuning ULPC parameters on a cell-by-cell basis, uniform settings (i.e., $P_{0}(i)=P_{0}(j)$ and $U_{U L}(i)=$ $\left.U_{U L}(j), \forall i, j\right)$ are also tested. In this case, a set of benchmarking solutions is constructed by selecting different combinations of $P_{0}$ and $U_{U L}$.

To assess the value of a parameter plan, two performance indicators are used: a) as a measure of network capacity, overall average user throughput, $\overline{T H_{\text {avg }}(i)}$,

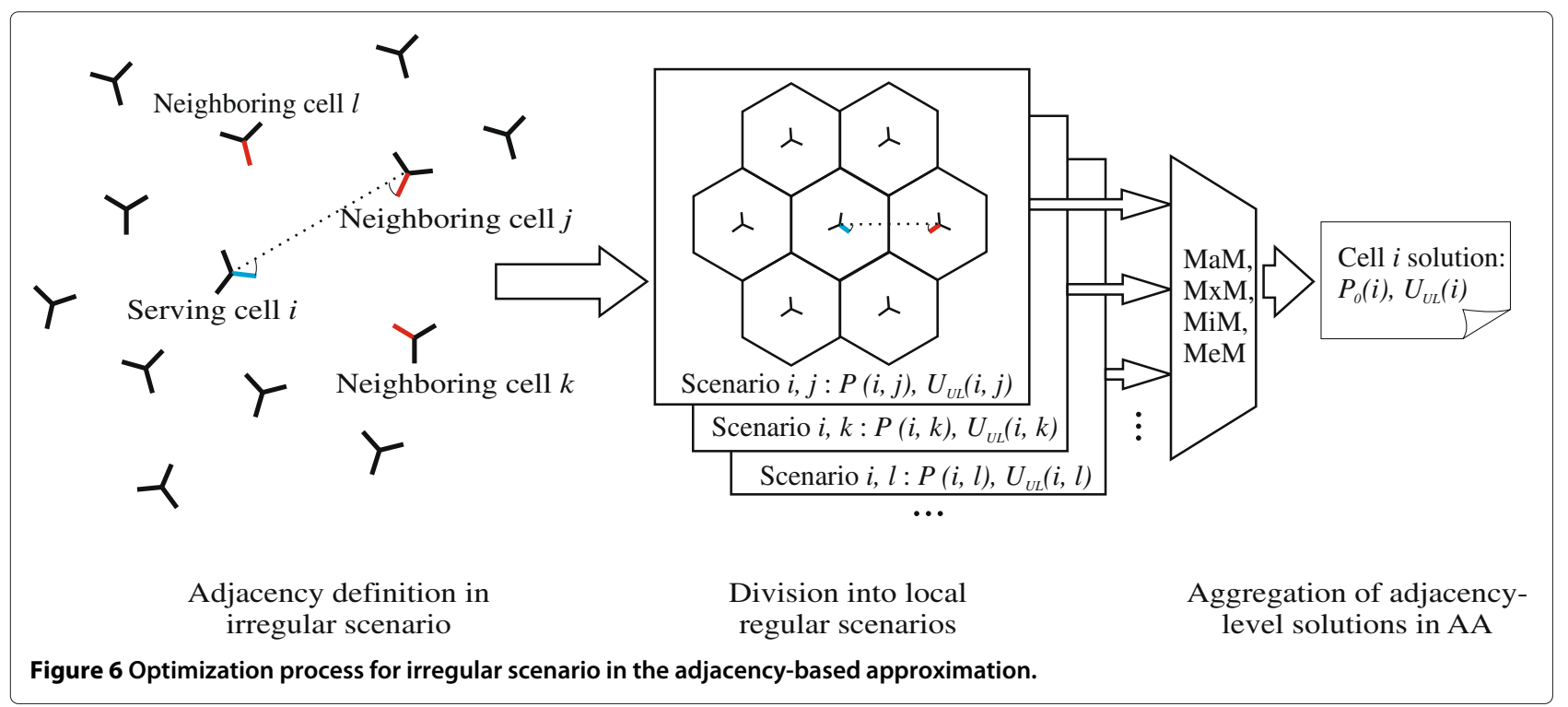


computed by averaging $T H_{\text {avg }}(i)$ across cells in the scenario, and b) as a measure of coverage, the ratio of cells fulfilling $T H_{c e}(i)>T H_{c e, m i n}$, denoted as $R_{T H_{c e, m i n}}$. In this work, $T H_{c e, \min }=100 \mathrm{kbps}$, based on typical operator demand.

\subsection{Results}

The analysis is first focused on network performance obtained with uniform parameter settings. Then, the analysis proceeds to cell-based solutions obtained by the proposed self-planning methods. Finally, execution time is analyzed.

\subsubsection{Uniform parameter settings}

Figure 7 shows the coverage-capacity trade-off achieved with uniform $P_{0}$ and $U_{U L}$ settings. Each point in the figure corresponds to a network parameter plan. Different curves correspond to different $U_{U L}$ values (i.e., $70 \%$, $80 \%, 90 \%$, and $100 \%$ ) and points in the curve correspond to $P_{0}$ values ranging from $-125 \mathrm{dBm}$ (down and left in the figure) to $-90 \mathrm{dBm}$ (up and left). Note that network performance is better in the upper right area of the figure. Four regions are defined in every curve, corresponding to different network behaviors, denoted as zone $1\left(P_{0} \geq-\right.$
$105 \mathrm{dBm})$, zone $2\left(P_{0} \in(-105,-115] \mathrm{dBm}\right)$, zone $3\left(P_{0} \in\right.$ $(-115,-119] \mathrm{dBm})$, and zone $4\left(P_{0}<-119 \mathrm{dBm}\right)$.

When $P_{0}$ is extremely high (zone 1 ), both coverage and capacity indicators improve as $P_{0}$ decreases. In this situation, interference levels are well above the noise floor, so decreasing $P_{0}$ in a cell and its neighbors decreases interference by the same amount. As a consequence, cell-edge throughput improves, while throughput for cell-center users is maintained as both interference and desired signal level decreases. When $P_{0}$ is medium (zone 2), a trade-off between coverage and capacity exists. Celledge throughput improves as $P_{0}$ further decreases at the expense of degrading network average throughput. Lower values of $P_{0}$ (zone 3 ) cause interference levels fall below the noise level, so that there is no benefit on further decreasing interference. On the contrary, cell-edge throughput starts to degrade. Finally, a strong degradation in network performance occurs when $P_{0}$ becomes less than the noise floor, $-119.4 \mathrm{dBm}$ in this work (zone 4).

The influence of $U_{U L}$ can be seen by comparing curves in Figure 7. It is observed that curves are shifted up and left when $U_{U L}$ increases. When more UL radio resources are available, users can reach higher throughput (i.e.,

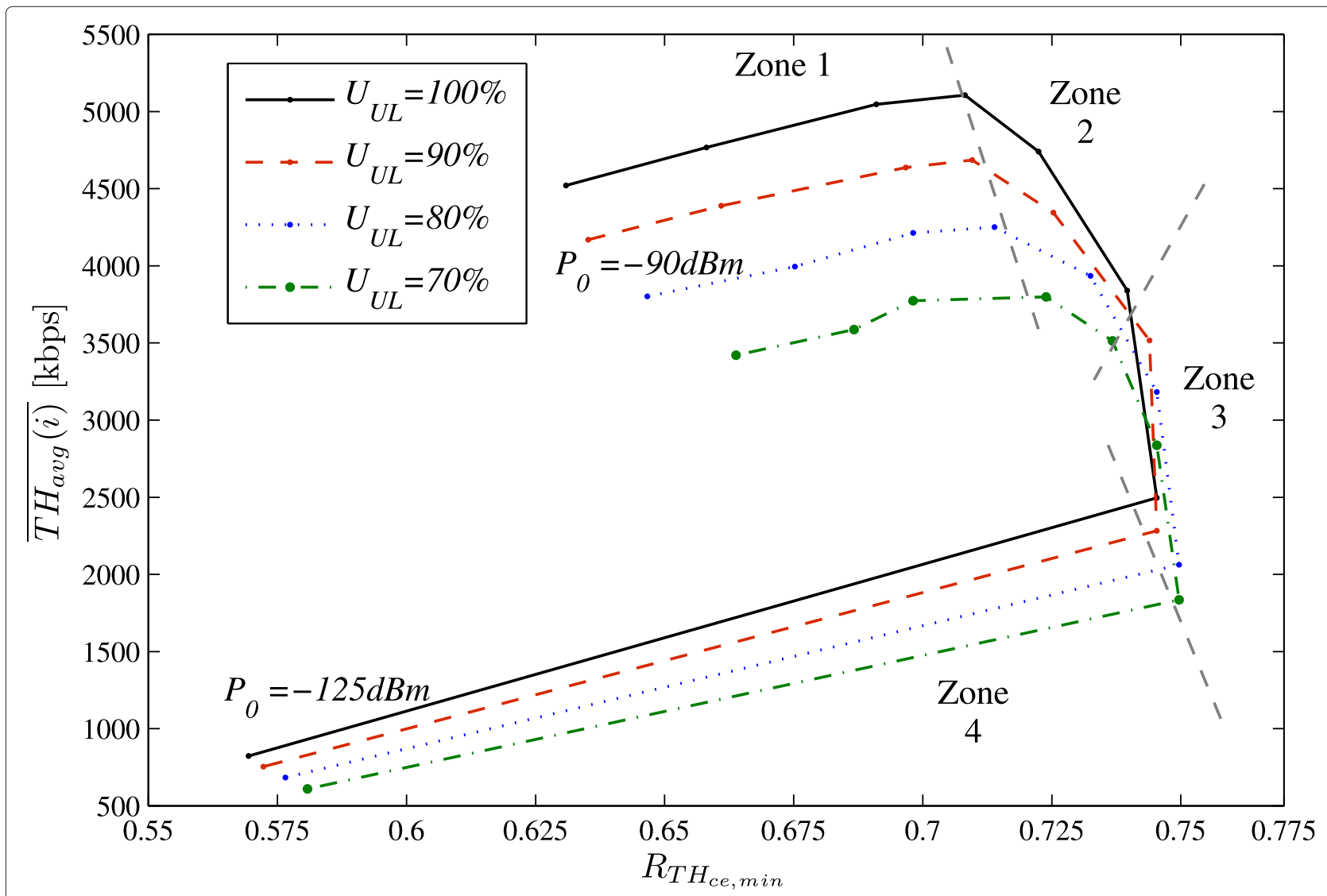

Figure 7 Overall network performance for uniform parameter settings. 
$\overline{T H_{a v g}(i)}$ increases), but interference levels also increase, and hence $R_{T H_{c e, m i n}}$ decreases.

\subsubsection{Non-uniform parameter settings}

Figure 8 compares the performance of the different self-planning proposals for cell-based ULPC parameter settings. For comparison purposes, results of uniform parameter plans are superimposed. Axis scales have been adjusted for optimal viewing in the figure. Note that MiM is not shown, since MiM experiences a very poor network performance (i.e., $\overline{T H_{\text {avg }}(i)}=1,254 \mathrm{kbps}$ and $R_{T H_{c e, \text { min }}}=$ $205 / 699=0.29$ ). This is due to the fact that MiM sets $P_{0}(i)$ to very low values, thus degrading SINR even for the best users. Other solutions (i.e., MaM, MeM, MxM, and MRA) perform similar to the best network performance with uniform settings (i.e., top-right in the figure). The best average throughput performance is achieved by MaM (i.e., $\overline{T H_{\text {avg }}(i)}=5,113 \mathrm{kbps}$ ), which outperforms MeM and MRA by $11.15 \%$ and $15.27 \%$, respectively. MaM selects the maximum value of $P_{0}$ and $U_{U L}$ across adjacencies, thus achieving better overall throughput. As a drawback, interference problems are greater, as pointed out by the lower value of $R_{T H_{c e, \min }}$ compared to other methods. The best cell-edge throughput performance is achieved by MeM (i.e., $R_{T H_{c e, \min }}=0.73$ ), which outperforms MaM and MxM by $3.87 \%$ and $2.04 \%$, respectively. Although MxM was designed as an intermediate solution between MaM and MiM, it is observed that limiting $U_{U L}$ in MxM has a strong impact on average user throughput, which is $19.89 \%$ and $11.09 \%$ lower than those achieved by MaM and MeM, respectively. MeM outperforms all other methods, as it is the only solution achieving a coverage-capacity tradeoff better than that obtained with any regular parameter setting.

\subsubsection{Execution time}

All methods were executed in an Intel ${ }^{\odot}$ Xeon $\odot$ computer with $3.47-\mathrm{GHz}$ clock frequency and $12 \mathrm{~GB}$ of RAM. The time required to build the curve for uniform $P_{0}$ and $U_{U L}$ settings in the static system-level simulator was $2,500 \mathrm{~s}$. In contrast, any of the self-planning methods proposed for constructing cell-based parameter plans took only $300 \mathrm{~s}$ in average (i.e., $0.43 \mathrm{~s}$ per cell).

It is also worth mentioning that, if a multi-variate exhaustive search approach is used, the addition of a new cell to an existing (i.e., already planned) scenario needs complete re-simulation of the whole scenario to build the new curve for uniform parameter settings. In

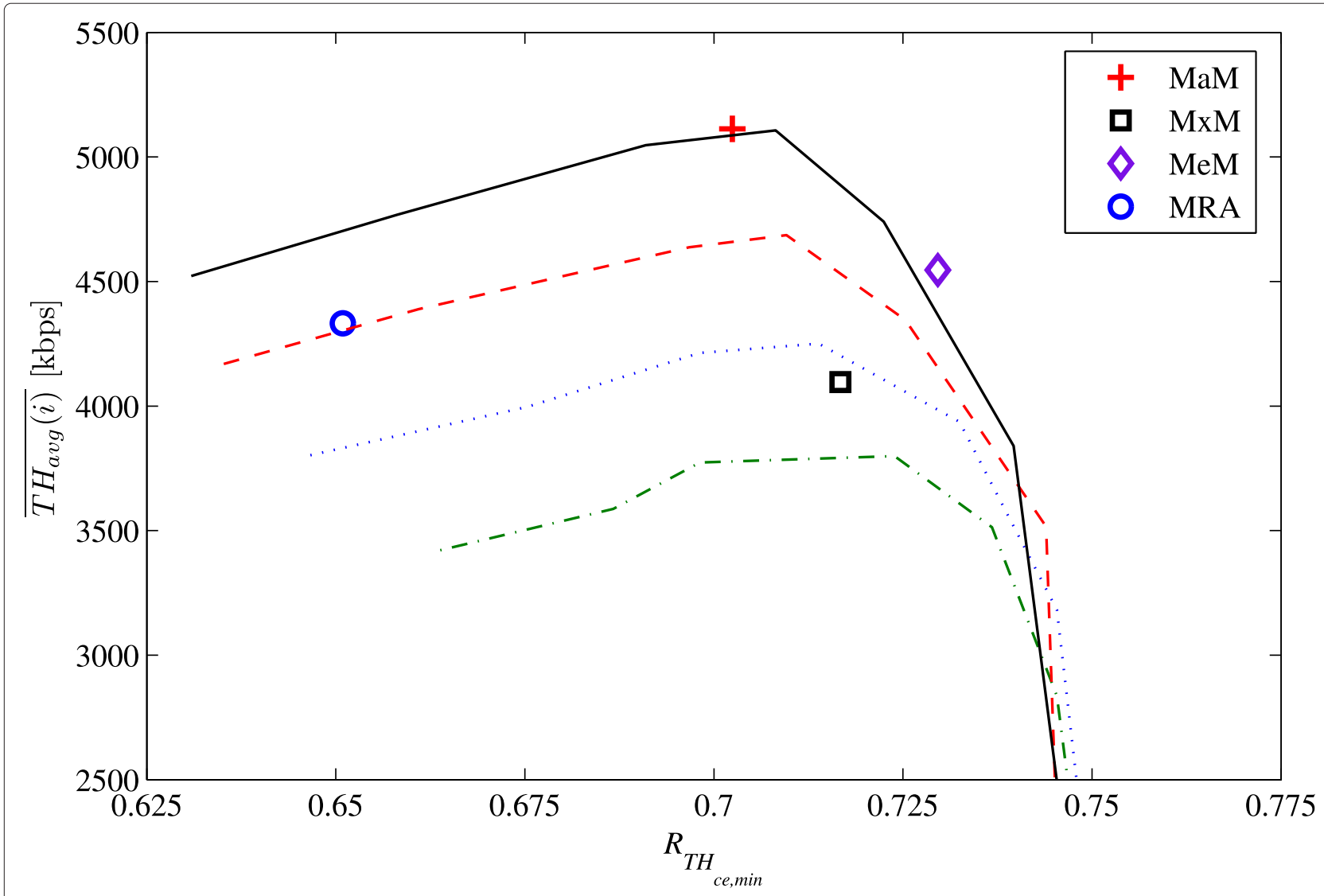

Figure 8 Overall network performance of self-planning methods. 
contrast, the self-planning method proposed in this work only needs solving $1+N_{\text {neigh }}(i)$ sub-problems (i.e., the recomputation of the new cell and its relevant neighbors) with regular scenarios.

\section{Conclusions}

In this work, a novel self-planning algorithm for UL power control parameters in the PUSCH of LTE has been presented. In the algorithm, the global network parameter optimization problem is divided into multiple simple optimization problems, one per adjacency, where a regular scenario is assumed. In such regular scenarios, a simple gradient descent method is used to find the optimal parameter settings that maximize average user throughput while still ensuring a minimum celledge user throughput. Then, the best parameter settings for each cell are calculated by aggregating adjacencylevel solutions. Thus, the proposed heuristic algorithm can deal with irregular scenarios at a low computational complexity, which is critical for planning large scenarios. Performance assessment has been carried out over a static system-level simulator implementing a real scenario. Results show that averaging solutions across adjacencies (i.e., MeM aggregation method) achieves the best trade-off between coverage and capacity, outperforming uniform network configurations for nominal power and UL cell load parameters.

The proposed self-planning algorithm can be implemented as part of a centralized SON system, which communicates with the network management system (NMS). For an initial operational network stage, self-configuration can be performed by automatically retrieving ULPC parameters from the NMS. Later, every time a new eNB is added, the network self-planning algorithm can be relaunched. For this purpose, the new eNB sends its location and antenna configuration parameters to the NMS, which updates network topology in the SON server. Then, the SON server computes the new ULPC parameter configuration, which is sent to NMS so that it can be retrieved by the new eNB.

\section{Competing interests}

The authors declare that they have no competing interests.

\section{Acknowledgements}

This work has been funded by the Spanish Ministry of Economy and Competitiveness (TIN2012-36455), Optimi-Ericsson and Agencia IDEA (Consejería de Ciencia, Innovación y Empresa, Junta de Andalucía, ref. 59288) and FEDER.

\footnotetext{
Author details

${ }^{1}$ Ingeniería de Comunicaciones, Universidad de Málaga, Campus de Teatinos S/N, 29071 Malaga, Spain. Ericsson, Via de los Poblados 13, 28033 Madrid, Spain
}

Received: 28 October 2014 Accepted: 4 March 2015

Published online: 20 March 2015

\section{References}

1. A Hoikkanen, in International Conference on Wireless and Optical Communications Networks, WOCN. Economics of 3G Long-Term Evolution: the business case for the mobile operator (Singapore, July 2007), pp. 1-5

2. F Gordejuela-Sanchez, J Zhang, in IEEE Global Telecommunications Conference, GLOBECOM. LTE Access network planning and optimization: A service-oriented and technology-specific perspective (Hilton Hawaiian Village, Honolulu, Hawaii, USA, December 2009), pp. 1-5

3. J Lempiainen, M Manninen, Radio Interface System Planning for GSM/GPRS/UMTS. (Springer, New York, NY, USA, 2001)

4. J Laiho, A Wacker, T Novosad, Radio Network Planning and Optimisation for UMTS. (John Wiley \& Sons, New York, NY, USA, 2002)

5. AR Mishra, Advanced Cellular Network Planning and Optimisation. (John Wiley \& Sons, New York, NY, USA, 2006)

6. 3GPP TS 32.500, Telecommunication management; Self-Organizing Networks (SON); Concepts and Requirements. V9, ETSI, Sophia Antipolis Cedex, France, December 2009

7. NGMN Use Cases Related to Self Organising Network, Overall description. http://www.ngmn.org. Accessed 1 October 2014

8. J Ramiro, K Hamied, Self-Organizing Networks: Self-Planning, Self-Optimization and Self-Healing for GSM, UMTS and LTE. (John Wiley \& Sons, New York, NY, USA, 2011)

9. S Hamalainen, H Sanneck, C Sartori, LTE Self-Organising Networks (SON). (John Wiley \& Sons, New York, NY, USA, 2011)

10. JF Whitehead, in IEEE 43rd Vehicular Technology Conference, VTC. Signal-level-based dynamic power control for co-channel interference management (Secaucus, New Jersey, USA, May 1993), pp. 499-502

11. 3GPP TS 36.213, Physical Layer Procedures. V8.6. ETSI, Sophia Antipolis Cedex, France, September 2009

12. W Xiao, R Ratasuk, A Ghosh, R Love, Y Sun, R Nory, in IEEE 64th Vehicular Technology Conference, VTC. Uplink power control, interference coordination and resource allocation for 3GPP E-UTRA (Montréal, Québec, Canada, September 2006), pp. 1-5

13. AM Rao, in IEEE 66th Vehicular Technology Conference, VTC. Reverse link power control for managing inter-cell interference in Orthogonal Multiple Access Systems (Baltimore, Maryland, USA, September 2007), pp. 1837-1841

14. A Simonsson, A Furuskar, in IEEE 68th Vehicular Technology Conference, VTC. Uplink Power Control in LTE - Overview and performance, Subtitle: Principles and benefits of utilizing rather than compensating for SINR variations (Calgary, Alberta, Canada, September 2008), pp. 1-5

15. B Muhammad, A Mohammed, in IEEE 70th Vehicular Technology Conference, VTC. Performance evaluation of uplink closed loop power control for LTE system (Anchorage, Alaska, USA, September 2009), pp. 1-5

16. B Muhammad, A Mohammed, in 6th International Conference on Emerging Technologies (ICET). Uplink closed loop power control for LTE system (Islamabad, Pakistan, October 2010), pp. 88-93

17. S Yang, Q Cui, X Huang, X Tao, in IEEE 72nd Vehicular Technology Conference, VTC. An effective uplink power control scheme in CoMP systems (Ottawa, Ontario, Canada, 2010), pp. 1-5

18. NJ Quintero, Advanced power control for UTRAN LTE uplink. PhD thesis, Department of Electronic Systems, Aalborg University, 2008

19. CU Castellanos, DL Villa, C Rosa, KI Pedersen, FD Calabrese, P-H Michaelsen, J Michel, in IEEE 67th Vehicular Technology Conference, VTC. Performance of uplink fractional power control in UTRAN LTE (Singapore, May 2008), pp. 2517-2521

20. C Suh, AT Koc, S Talwar, in IEEE Global Telecommunications Conference, GLOBECOM. Trade-off power control for cellular systems (Honolulu, Hawaii, USA, November 2009), pp. 1-6

21. S Xu, M Hou, K Niu, Z-Q He, W-L Wu, Coverage and capacity optimization in LTE network based on non-cooperative games. J. China Univ Posts Telecommunications. 19(4), 14-42 (2012)

22. A Awada, B Wegmann, I Viering, A Klein, Optimizing the radio network parameters of the Long Term Evolution system using Taguchi's method. IEEE Trans. Vehicular Technol. 60(8), 3825-3839 (2011)

23. K Majewski, M Koonert, Analytic uplink cell load approximation for planning Fractional Power Control in LTE networks. Telecommun. Syst. 52(2), 1081-1090 (2013)

24. CU Castellanos, FD Calabrese, KI Pedersen, C Rosa, in IEEE 68th Vehicular Technology Conference, VTC. Uplink interference control in UTRAN LTE 
based on the Overload Indicator (Calgary, Alberta, Canada, September 2008), pp. 1-5

25. 3GPP R1-074349, Overload Indicator handling for LTE, TSG RAN WG1 \#50bis Meeting. V8.6, ETSI, Sophia Antipolis Cedex, France, September 2007

26. M Dirani, Z Altman, Self-Organizing Networks in next generation radio access networks: Application to Fractional Power Control. Comput. Netw. 55(2), 431-438 (2011)

27. LTE; Evolved Universal Terrestrial Radio Access (E-UTRA); Long Term Evolution (LTE) Physical Layer; General Description. V8.3, ETSI, Sophia Antipolis Cedex, France, April 2009

28. S Sesia, I Toufik, M Baker, LTE, the UMTS Long Term Evolution: from Theory to Practice. (Wiley, New York, NY, USA, 2009)

29. 3GPP TR 36.942, LTE; Evolved Universal Terrestrial Radio Access (E-UTRA); Radio Frequency (RF) System Scenarios. V8.2, ETSI, Sophia Antipolis Cedex, France, July 2009

30. 3GPP TS 32.521, Technical Specification Group Services and System Aspects; Telecommunications Management; Self-Organizing Networks (SON) Policy Network Resource Model (NRM) Integration Reference Point (IRP); Requirements. V9, ETSI, Sophia Antipolis Cedex, France, March 2010

31. S Louvros, K Aggelis, A Baltagiannis, in Proceedings of the 11th International Conference on Telecommunications (CONTEL). LTE cell coverage planning algorithm optimising uplink user cell throughput (Graz, Austria, June 2011), pp. 51-58

32. 3GPP TR 36.814, Technical Specification Group Radio Access Network: Evolved Universal Terrestrial Radio Access (E-UTRA); Further Advancements for E-UTRA Physical Layer Aspects. V9, ETSI, Sophia Antipolis Cedex, France, March 2010

\section{Submit your manuscript to a SpringerOpen ${ }^{\circ}$ journal and benefit from:}

- Convenient online submission

- Rigorous peer review

- Immediate publication on acceptance

- Open access: articles freely available online

- High visibility within the field

- Retaining the copyright to your article 